\title{
A Clinicopathological Appraisal of Port-site Infections after Laparoscopic Cholecystectomy: Our Experience at Shaheed Suhrawardy Medical College Hospital
}

\author{
Khan RK ${ }^{1}$, Rahman MM², Ahmed N³, Sarker RD ${ }^{4}$
}

Conflict of Interest: None

Received: $03-08-2020$

Accepted: $27-10-2020$

www.banglajol.info/index.php/JSSMC

\section{Key Words:}

Port site infection, Laparoscopic cholecystectomy, Cholecystitis, TB, Sinogram.

\begin{abstract}
Background: Undoubtedly the port site infections have remarkably enhanced to be a stigma as post laparoscopic morbidity and is still a field of debate and further exploration to surgeons. These port site infections (PSI) are infrequent surgical site infection, complicating the recovery of patients undergoing laparoscopic cholecystectomy. Hence, the necessity of further evaluation of the regarded facts was intended to be explored.
\end{abstract}

Aim: This study was aimed to evaluate the factors that created or provoked PSI after laparoscopic removals of gall-bladder and at analysing which of these factors can be traced and modified to combat PSI in a trail to conquer these complications and to achieve maximum advantages from laparoscopic surgeries.

Methods: A prospective descriptive qualitative study conducted on patients who underwent laparoscopic cholecystectomies in our hospital as well as at other facilities remote from our work stations. Factors as gender, site of infected port, types of microorganism, acuteness versus chronicity of the disease, types of infection (superficial or deep infection) and intraoperative spillage of stones, bile or pus were analysed in our study. Swabs were taken for culture and sensitivity tests in all patients who developed infections. Explorations were done under GA for some patients who had deep surgical site infections and wound debridement was done, excisional biopsies were taken for histopathological studies, and tissue samples for Gene-Xpert analysis for detection of Mycobacterium tuberculosis was done. All patients were followed up for six months postoperatively at our surgery unit rooms.

Results: Port site infection rate was recorded in 40 cases amongst 340 procedures from the July 2018 to June 2020 (11.76\%). A higher rate was observed in female patients 32 cases (9.41\%) and 13 cases (3.82\%) of acute cholecystitis. Larger number of cases of the PSI were superficial infections (77.5\%) with non-specific microorganism in 34 cases $(80.0 \%)$.

Conclusion: We reconciled a significant association of PSI with spillage of bile or stones during the procedure and with acute cholecystitis. Most of the infections are superficial and more common in males. Precautions and protocols should be taken in measure to avoid unnecessary hurry and faulty procedure of sterilization during and prior to the whole surgery to combat PSI effectively.

[J Shaheed Suhrawardy Med Coll 2020; 12(2): 76-81] DOI: https://doi.org/10.3329/jssmc.v12i2.56886

1. Dr. Rafiul Karim Khan, Assistant Professor, Department of Surgery, Shaheed Suhrawardy Medical College, Dhaka.

2. Professor Dr. Md. Mustafizur Rahman, Professor and HOD, Department of Surgery, Shaheed Suhrawardy Medical College, Dhaka.

3. Dr. Nadim Ahmed, Senior Consultant, Department of Surgery, Shaheed Suhrawardy Medical College Hospital, Dhaka

4. Dr. Rajib Dey Sarker, Assistant Registrar, Department of Surgery, Shaheed Suhrawardy Medical College Hospital, Dhaka.

Address of Correspondence: Dr. Rafiul Karim Khan, Assistant Professor, Department of Surgery, Shaheed Suhrawardy Medical College Hospital, Dhaka, Bangladesh. Mobile: +8801752-477745, Email: rafiulronyaz@gmail.com,

\section{Introduction}

To be precise, post-operative infections are a point of agony for any surgeon around the world and a surgical team usually leaves no stone unturned to prevent such unwanted hazard. This theme is more notorious in minimal access surgery (MAS) as the benefit of which is to give less hospital stay and less post-operative discomfort to patient. But port site infections in its increasing statistics have been reported ${ }^{1,2}$ to be severe enough to indulge patient's safety and satisfaction. However, the treatment 
protocol, including the drug regimen and duration of therapy varied from surgeon to surgeon and from hospital to hospital.

Laparoscopic surgery also termed as key-hole surgery minimally invasive surgery, was first presented in the beginning of $20^{\text {th }}$ century, and then it eventually became the surgical treatment of choice for many surgeries. ${ }^{3}$ Undoubtedly laparoscopic cholecystectomy (Lap. Chole.) is the gold standard treatment for symptomatic gallstone disease. ${ }^{4}$ Its advantages include decreased hospital stay postoperatively, earlier return to daily work, minimum postoperative pain, ${ }^{5}$ small surgical incisions and so better cosmetic outcomes and minimal postoperative complications. So, it not only overran open cholecystectomy but also more or less ended attempts for the noninvasive management of gallstones, such extracorporeal shock wave, and bile salt therapy. ${ }^{6}$ One of surgical procedure complications is surgical site infection (SSI). Infection could be intrinsic and/or extrinsic as the human body enables the survival of a wide variety of microorganisms with potential for infection as a result of surgical intervention. ${ }^{7}$ Patient's bacterial flora may become opportunistic and cause infection in special circumstances. This can occur in both open surgeries and to a lesser extent in laparoscopic one. ${ }^{8}$ It is a fact that laparoscopic cholecystectomy associated with fewer SSI than open cholecystectomy. ${ }^{9}$ However now-a-days, with increasing number of performed laparoscopic cholecystectomies, there is an increasing number of port site infection (PSI).

Although it occurs infrequently, but it has significant influence on overall outcomes of laparoscopic cholecystectomy and its final results like delay in return to work, increase cost and bad cosmetic results which become disappointing for both patient and the surgeon. There are three types of surgical site infection which can occur in port site: ${ }^{10-12}$ First is superficial surgical site infection occurring within 30 days post-surgery and involves only skin and subcutaneous tissues and the patient at least has one of the following: (a) purulent discharge from the superficial incision, (b) organism isolated from aseptically obtained culture of fluid or tissue from superficial incision. The second type is deep surgical site infection which may be presented after 30 days of operation and involves deep soft tissues including fascia and muscles deep to the incision. The patient has at least two of the followings: a) purulent drainage from deep incision, b) dehiscence of the deep incision, and c) an abscess. The third type is organ/space SSI where infection involves any organ and spaces other than the incision which was opened or manipulated during surgery. ${ }^{13}$

\section{Patients and methods}

In this prospective descriptive qualitative study conducted at Shaheed Suhrawardy Medical College Hospital, Sher-e-Bangla Nagar, Dhaka, Bangladesh, from 1st of July 2018 to 30th of June 2020 (24 months) period, patients who underwent laparoscopic cholecystectomies in the same facility or any other referral facility connected with same government line-ups were included in our study. All the cases were given 1 dose of 1 gram of Ceftriaxone and 1 dose of $100 \mathrm{ml}$ of Metronidazole preoperatively. This antibiotic combination was carried out at least up to $3^{\text {rd }}$ post-operative day. All operations were done by experienced surgeons, using four ports procedures, with reusable instruments; gallbladder was extracted from the epigastric port in all operations, without using endobag. Sub-hepatic tube drain was used in most of the patients and removed next day after the operation. Stitches were removed 7th post-operative day without the presence of infection. Swabs were taken for culture and sensitivity in all patients who developed PSI. Exploration under general anaesthesia was done for patients with chronic deep site infections, presented with persistent discharging sinus and after doing sinogram. Wound debridement was done and the wound was left open to heal by secondary intention. Excisional biopsies for chronic discharging sinuses had been done and sent for histopathological studies and tissue samples examined with 'Gene Xpert" for Mycobacterium tuberculosis. In patients proved to have TB, anti TB-therapy was given orally.

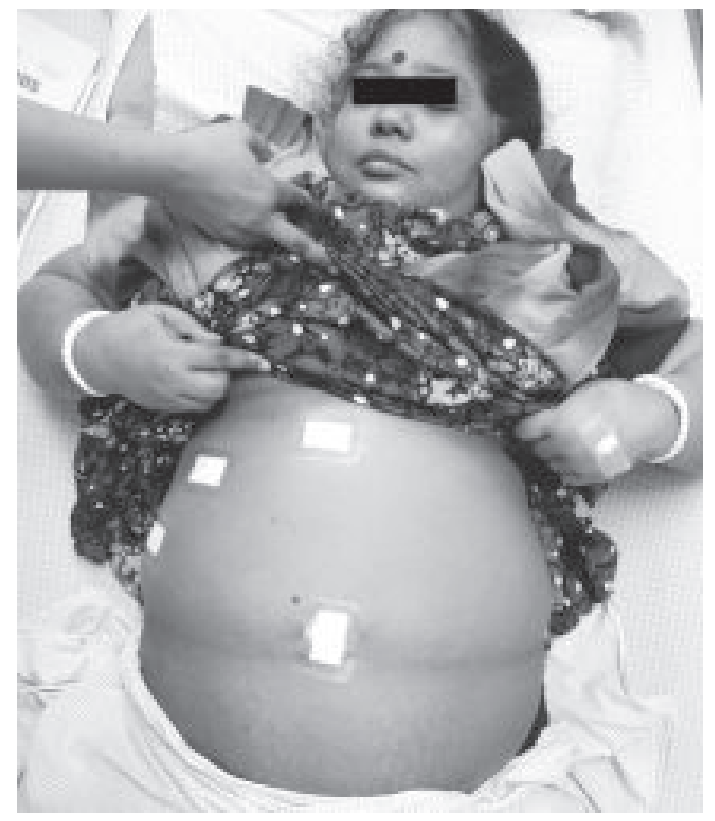

Fig.-1: Image of port positions of a 48-year old female patient at $4^{\text {th }}$ POD after Lap. Chole. who developed PSI on $8^{\text {th }} P O D$ 


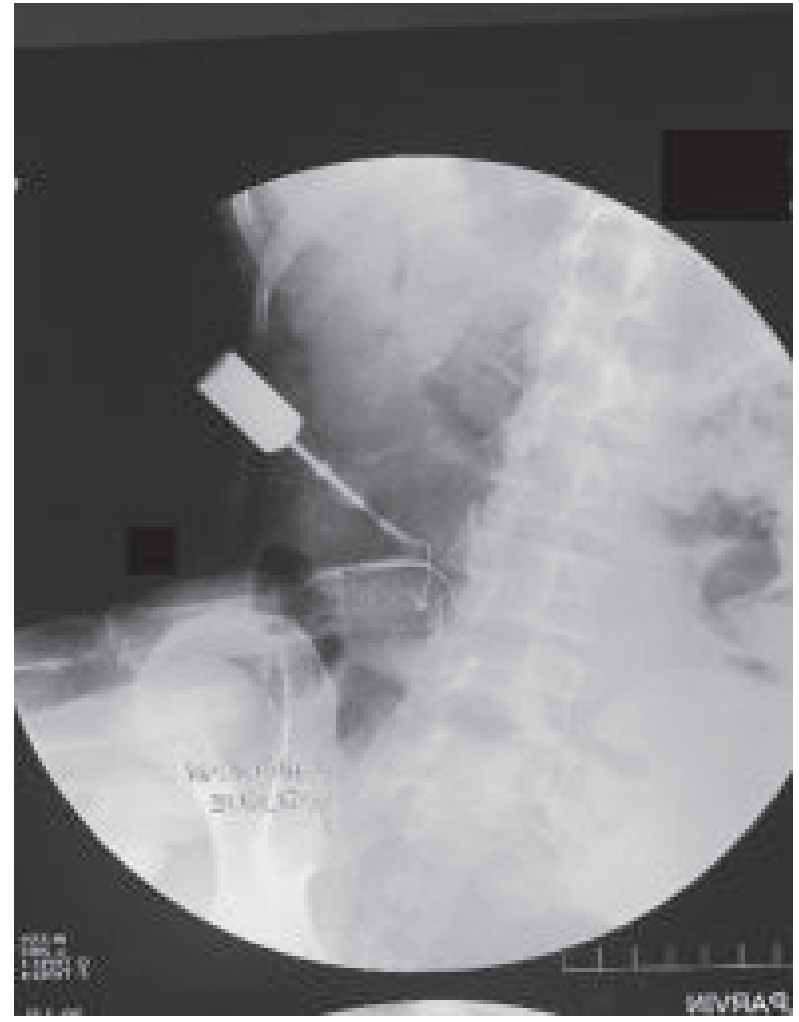

Fig.-2: Sinogram images of port-site sinus tract

All patients responded well within six months of followup. Patient's whose operations were converted to open procedures and those with a history of chronic co-morbid conditions were excluded from the sample to minimize bias in the study. For the same reason, and to avoid iatrogenic complications of beginner surgeons, we collect the data of experienced surgeons who have good documentation and postoperative follow-up. Factors as gender, site of infected port, type of microorganism, acute versus chronic cholecystitis, type of infection (superficial or deep infection) and intraoperative spillage of stones, bile or pus were analysed in our sample. The method of sterilization used in our sample was washed the instruments by hydrogen peroxide solution, then rinse with tap water, finally emersion in or Cidex ${ }^{\circledR}$ solution for 60 minutes.

\section{Results}

We included 340 patients in our study based on the inclusion criteria narrated earlier. Their ages ranged from 20 to 65 years (mean age 43.1 years), PSI was found in 40 / $11.76 \% 340$ patients (4.5\%). Regarding gender, in 32/340 female patients, percentage of the PSI was $9.41 \%$ and in $8 /$ 340 male patients the percentage was $2.35 \%$. There is an association between female gender and infection, $\mathrm{p}$-value 0.03. as in Table I.
Table-I

The incidence of PSI in relation to gender

\begin{tabular}{lccc} 
Gender & Infected & Percentage & P value \\
\hline Male & 8 & $2.35 \%$ & 0.03 \\
Female & 32 & $9.41 \%$ & \\
\hline Total & 40 & - & \\
\hline
\end{tabular}

Regarding the status of gallbladder before the operation, 96 patients $(28.2 \%)$ were operated during an acute attack and 244 patients $(71.6 \%)$ were suffering from chronic cholecystitis. P value was 0.001 , i.e., there is a significant association between infection and acute cholecystitis as shown in Table II.

\section{Table-II}

Incidence of PSI in relation to the clinical diagnosis of the gallbladder pre-operatively

\begin{tabular}{lccc} 
Condition & $\begin{array}{c}\text { Infected / } \\
\text { Total Subjects }\end{array}$ & Percentage & $\begin{array}{c}\mathrm{P} \\
\text { Value }\end{array}$ \\
\hline Chronic case & $13 / 244$ & $5.32 \%$ & 0.001 \\
Acute case & $27 / 96$ & $28.13 \%$ & \\
\hline Total & $40 / 340$ & - & \\
\hline
\end{tabular}

Concerning spillage of bile, stones, or pus, 24/80 patients (30.0\%) developed infection while spillage occurred during their operations and $16 / 260$ patients $(6.15 \%)$ developed infection despite no spillage occurred. P value was 0.0001 i.e., the spillage can be regarded as a risk factor in the development of PSI (Table III).

\section{Table III}

Incidence of PSI in relation to spillage of bile, stones, or pus during operation

\begin{tabular}{lccr}
$\begin{array}{l}\text { Incident of } \\
\text { spillage }\end{array}$ & $\begin{array}{c}\text { Infected / } \\
\text { Total Subjects }\end{array}$ & Percentage & $\begin{array}{c}\mathrm{P} \\
\text { Value }\end{array}$ \\
\hline With spillage & $24 / 80$ & $30.00 \%$ & 0.0001 \\
Without spillage & $16 / 260$ & $6.15 \%$ & \\
\hline Total & $40 / 340$ & - & \\
\hline
\end{tabular}

According to the site of port infection, 32 patients (80.0\%) developed an infection at the epigastric port, 06 patients $(15.0 \%)$ developed an infection at the umbilical port and only 02 patients $(5.0 \%)$ developed an infection at the lateral ports. P value was 0.0001 , which is highly significant and site of gallbladder extraction could be a cause of PSI (Table IV). 
Table IV

\begin{tabular}{lccc}
\multicolumn{3}{c}{ Incidence of PSI in different port sites } \\
Port Positions & $\begin{array}{c}\text { Infected / } \\
\text { Total Subjects }\end{array}$ & Percentage & $\begin{array}{c}\mathrm{P} \\
\text { Value }\end{array}$ \\
\hline Umbilical Port & $32 / 40$ & $80.0 \%$ & 0.0001 \\
Epigastric Port & $06 / 40$ & $15.0 \%$ & \\
Lateral Ports & $02 / 40$ & $5.0 \%$ & \\
\hline Total & $40 / 340$ & - & \\
\hline
\end{tabular}

Regarding the type of port site infection, $31 / 40$ patients (77.5\%) developed a superficial infection and $9 / 40$ patients (22.5\%) developed deep site infection as in Table V.
Table V

\begin{tabular}{lccc}
\multicolumn{4}{c}{ Types of Port Site Infection } \\
Types of PSI & $\begin{array}{c}\text { Infected / } \\
\text { Total Subjects }\end{array}$ & Percentage & $\begin{array}{c}\mathrm{P} \\
\text { Value }\end{array}$ \\
\hline Superficial PSI & $31 / 40$ & $77.5 \%$ & 0.0001 \\
Deep PSI & $09 / 40$ & $22.5 \%$ & \\
\hline Total & $40 / 340$ & - & \\
\hline
\end{tabular}

About results of swabs culture and PCR of tissue samples, 18 patients $(45 \%)$ were infected by Gram (-ve) bacteria, 10 patients $(25 \%)$ were infected by Gram (+ve) bacteria, 3 patients $(7.5 \%)$ were infected with mixed infection, 03 patients $(7.5 \%)$ whose results were chronic inflammation with no growth and six patients (15\%) were infected by Mycobacterium species, Table VI.

Table VI

\begin{tabular}{llcc} 
& Type of microorganism associated with post laparoscopic cholecystectomy \\
Types & Microorganism & No of Cases & Percentage \\
\hline Non-specific infection & & 34 & $85 \%$ \\
Gram Negative & Enterobacter spp. & 14 & $35 \%$ \\
& E.coli & 10 & $25 \%$ \\
Gram Positive & Staphylococcus & 08 & $20 \%$ \\
& Enterococcus & 02 & $05 \%$ \\
Mixed & & 03 & $7.5 \%$ \\
No growth & & 03 & $7.5 \%$ \\
Specific infection & Mycobacterium & $06 / 40$ & $15 \%$ \\
& Atypical & 03 & $7.50 \%$ \\
\hline
\end{tabular}

We also encountered patients with discharging sinus from ports and did sinogram to rule out any other pathologies intraperitoneally. $07(17.5 \%)$ cases went for sinogram and amongst them 05 (12.5\%) cases revealed an inflamed sinus tract at port site. So there is trifle association of PSI with port site sinus tract, narrated in Table VII.

\section{Table VII}

\section{Port site sinus tract formation with PSI}

\begin{tabular}{lcc} 
Presence of Sinus Tract & No of Cases & Percentage \\
\hline No sinus tract & 33 & $82.5 \%$ \\
Discharging sinus tract & 07 & \\
Sinogram attempts & 07 & $17.5 \%$ \\
Positive Sinogram & 05 & $12.5 \%$ \\
\hline
\end{tabular}

\section{Discussion}

Surgeons from different parts of the world are still combating the stigma of PSI after cholecystectomy. Patients are influentially adopting the minimal invasive procedures as their choice of surgery. They wish to get maximum cosmetic benefit as well as least suffering of pain and discomfort of hospital staying. But these incidences of PSI are getting truly bizarre in some cases for both patient and his surgeon. Still the incidences of PSI after laparoscopic cholecystectomy are lower than that of open cholecystectomy because laparoscopic procedures are minimally invasive technique and have less impact on the immune system than an open one. ${ }^{\mathbf{1 4}}$

The incidence of port site infection in our sample is about $11.76 \%$ (40 patients from 340 ) which was remarkably higher than results of study done by Khurshid, et al. in Indian 
hospital of Kashmir in 2012, their results was $6.7 \%{ }^{\mathbf{1 5}}$ and by Jasim Saud, et al. which performed in AL Basrah General Hospital 2010 that was $2.4 \%{ }^{16}$

We found the majority of patients underwent laparoscopic cholecystectomy were females ( 280 patients amongst 340 , $73.68 \%$ ), also most of our port site infection patients were females 32 patients from 40 patients. If we compare with another study that was done in Al-Basrah Hospital which included 369 patients, 301 (81.57\%) of them were females and $68(18.43 \%)$ were males and PSI occur in 11 patients (2.98\%), 7 females (63.63\%) and 4 males (36.36\%). ${ }^{14}$ In both studies, although number of female is higher than males) and perforation of gallbladder during operation more in males than in females. ${ }^{17}$ From a total number of 340 patients, we found 60 patients had chronic cholecystitis before operation and 280 were operated during the acute phase, $27 / 96$ patients (28.13\%) were operated during the chronic phase of cholecystitis and developed PSI and 13/ 244 patients $(5.32 \%)$ were operated during acute inflammation and got infected. i.e., infection was more commonly affecting patients during acute phase than with chronic phase. It's $p=0.001$. In comparing with other study done in DHQ (Divisional Headquarters Teaching Hospital) Mirpur-Kashmir show $7.1 \%$ PSI in their sample, $65 \%$ of cases were during the acute phase and $35 \%$ were in chronic cases. ${ }^{9}$ Both studies show the significance of acute phase with PSI. This is due to increased probability of perforation of gallbladder and spillage of bile, stones, or pus as a result of difficult manipulation, tensely distended gallbladder with thickened oedematous wall. ${ }^{18}$ As long as the inflammation is limited to gallbladder, laparoscopic cholecystectomy is usually feasible. Great care must be taken in proceeding with operations if the inflammation reaches porta hepatis, as normally thin minimally adhesive tissue that invest cystic duct and artery is markedly thickened and oedematous and may not readily separated by usual blind dissection with a clean-cut CVS. ${ }^{19}$

The surgery may also be associated with spillage of gallstones in 5\% to $40 \%{ }^{13,19}$ of procedures and perforation of gallbladder during surgery occur frequently at a remarkable rate $(10 \% \text { to } 40 \%)^{20}$ and may occur secondary to traction applied by grasping forceps or because of electro-surgical thermal injury during removal of the gallbladder from its fossa. ${ }^{18}$ Spillage of bile, pus or stones which can be retained inside the abdomen or in the wound is highly associated with port site infection and abscess formation ${ }^{18}$ which was statistically significant $(\mathrm{p}=0.0001)$. Foreign body retained could be stones, clips, or parts of plastic sheath. Another study done in Taj Surgery Hospital in Pakistan for three years 2009-2012 show relation between port site infection and intraoperative spillage during laparoscopic cholecystectomy in $5.3 \%$ of perforated cases. ${ }^{21}$

This study was done in governmental medical college hospital in Dhaka (Capital), Bangladesh which is alos a referral institute and receives several cases from hospitals of periphery and those cases were also taken in consideration. Another data in in a medical college of India shows high association between epigastric port and infection $(88.2 \%)^{22}$ and in another study, shows surgical site infection in umbilical port more than epigastric port and this related to umbilical flora and gall bladder extraction through umbilicus in single port surgery ${ }^{21}$ which indicates that site of gall bladder extraction was the most common site of PSI. Most of the patients presented with PSI in our study were superficial infection $31 / 40$ patients $(77.5 \%)$ compared with $9 / 40$ patients $(22.5 \%)$ presented with deep site infection. Also, superficial infection is more common than deep infection as reported by study done by Mir, et al. at tertiary care hospital of Kashmir 2012 (87.7\% for superficial infection compared with $13.3 \%$ for deep infection). ${ }^{15} 09$ patients $(22.5 \%$ ) who presented with deep infection in our study as recurrent discharging single or multiple sinuses that was also evidenced with sinogram study with contrast (07 at epigastric port).

Three of these were infected with atypical mycobacterium species, three were infected with typical mycobacterium tuberculosis, one patient had retained stone in deep layers where infection was mixed, other patient had retained foreign body (plastic sheath of a laparoscopic instrument) inside deep layers of falciform ligament where no growth of bacteria was obtained and only chronic granulomatous reaction with chronic inflammatory cells was found and a patient had abscesses deep in the abdominal wall between epigastric and lateral port which were sterile. In our study, the percentage was higher maybe due to lack of usage of retrieval bag which prevent direct contact of port wound with the content of infected gallbladder. Port site infection was noticed in 32 patients (80\%) in epigastric port and six patients $(15 \%)$ in umbilical port and only two patients $(5 \%)$ at the lateral port $(\mathrm{p}=0.0001)$, which is statistically significant for the association between epigastric port and SSI. This may be due to the fact that the epigastric port is the site of gallbladder extraction therefore this port will be in direct contact with inflamed gallbladder.

By taking detailed history, one of the patients infected with mycobacterium species has close relative (her son in-law who was changing her dressing) working in hospital of infectious disease (250 Bed TB Hospital, Shyamoli, Dhaka). There is another study for the source of mycobacterium is the use of tap water for rinsing laparoscopic instruments after complete sterilization to 
rinse glutaraldehyde may re-introduce mycobacterium ${ }^{24}$ to the instrument and then to the wound. Also sharing of laparoscopic instruments with other department like urology has observed as another source of infection sometimes. ${ }^{25}$ The instruments itself covered by plastic insulation and presence of joints make its sterilization insufficient. $^{26}$

We also noticed huge loads of patient who were awaiting laparoscopic procedures and that undoubtedly compromised the optimum time for sterilization at our facility. In advanced centres, the golden standard is to use a disposable laparoscopic instrument, use of advanced sterilization methods such as (STERRAD) which is a trademark for low-temperature sterilization system, using gas plasma technology, quick, safe and efficient elimination of toxic residue from devices. ${ }^{27}$

\section{Conclusion}

Being a surgeon, we cannot overlook and cast away the stigma of PSI whatever the procedure or number of patients are. So, at the end of this study we would like to state that there is a significant association of PSI with spillage of bile, stones, or pus, with the port of gallbladder extraction and with acute cholecystitis. Special consideration should be taken in chronic deep surgical site infection as Mycobacterium tuberculosis could be the cause. Most of the PSIs are superficial and more common in females.

\section{Ethical Ground}

Due written permission was taken from patient or party prior to this study regarding treatment, imaging, archiving, research and publication.

\section{References}

1. Mukherjee R, et al. "A Critical Appraisal of Port Site Infections: Meta-Analysis of Existing Contemporary Data", Ramanuj Mukherjee, Shreya Sengupta*, and Sayantan Bose, Journal of Surgery \& Transplantation Science, 6(1): 1065.

2. Al-Naser M K H, "Port Site Infections After Laparoscopic Cholecystectomy", International Journal of Medical Research \& Health Sciences, 2017, 6(6): 132-13

3. Dubois, Francois, et al. "Laparoscopic cholecystectomy: Preliminary report of 36 cases." Annals of Surgery 211.1 (1990): 60-62.

4. Mehraj, Adnan, et al. "Laparoscopic cholecystectomy: An audit of 500 patients." Journal of Ayub Medical College Abbottabad 23.4 (2011): 88-90.

5. Williams, Lester F., et al. "Comparison of laparoscopic cholecystectomy with open cholecystectomy in a single center." The American Journal of Surgery 165.4 (1993): 459-465.

6. Pham, Thai H \& John G Hunter. Ch 32: Gallbladder and extrahepatic biliary system. Andersen, Dana K., et al., eds. Schwartz's Principles of Surgery. 10th ed. MC Graw Hill Education, 2015, P 1324.

7. Shindholimath, V.V., et al. "Factors influencing wound infection following laparoscopic cholecystectomy." Tropical Gastroenterology: Official Journal of the Digestive Diseases Foundation 24.2 (2002): 90-92.
8. Richards, Michael J., et al. "Nosocomial infections in combined medical-surgical intensive care units in the United States." Infection Control \& Hospital Epidemiology 21.08 (2000): 510-515.

9. Jan, Waqar Alam, et al. "The frequency of port-site infection in laparoscopic cholecystectomies." Journal of Postgraduate Medical Institute (Peshawar-Pakistan) 22.1 (2011)

10. Bhat, Sriram. SRB's Manual of Surgery. JP Medical Ltd, 2016.

11. Horan, Teresa C., et al. "CDC definitions of nosocomial surgical site infections, 1992: a modification of CDC definitions of surgical wound infections." American Journal of Infection Control 20.5 (1992): 271-274.

12. Williams, Norman S., et al. Bailey \& Love's Short Practice of Surgery. CRC Press, 2008.

13. Sathesh-Kumar, T., et al. "Spilled gall stones during laparoscopic cholecystectomy: a review of the literature." Postgraduate Medical Journal 80.940 (2004): 77-79.

14. Holub, Z. "Impact of laparoscopic surgery on immune function." Clinical and Experimental Obstetrics \& Gynecology 29.2 (2002): 77-81.

15. Mir M, S Khursheed, U Malik, B Bali. "Frequency and risk factor assessment of port-site infection after elective laparoscopic cholecystectomy in low-risk patients at a tertiary care hospital of Kashmir." The Internet Journal of Surgery Volume 28.2 (2012).

16. Jasim D saud and Mushtaq Ch Abu Al-Hail. "Surgical site infection after laparoscopic cholecystectomy." Basrah Journal of Surgery 16 (2010): 119-121.

17. Rice, David C., et al. "Long-term consequences of intraoperative spillage of bile and gallstones during laparoscopic cholecystectomy." Journal of Gastrointestinal Surgery 1.1 (1997): 85-91.

18. Zinner, Michael J. Maingot's abdominal operations. McGrawHill Publications, 2009. p 1004-1006.

19. Memon, M.A., et al. "The outcome of unretrieved gallstones in the peritoneal cavity during laparoscopic cholecystectomy." Surgical Endoscopy 13.9 (1999): 848-857.

20. Brockmann, J.G., et al. "Complications due to gallstones lost during laparoscopic cholecystectomy." Surgical Endoscopy 16.8 (2002): 1226-1232.

21. Taj, Muhammad Naeem, Yasmeen Iqbal, and Zakia Akbar. "Frequency and prevention of laparoscopic port site infection.” J Ayub Med Coll Abbottabad 24 (2012): 197-199.

22. Karthik, Somu, et al. "Analysis of laparoscopic port site complications: A descriptive study." Journal of Minimal Access Surgery 9.2 (2013): 59.

23. Mayol, Julio, et al. "Risks of the minimal access approach for laparoscopic surgery: multivariate analysis of morbidity related to umbilical trocar insertion." World Journal of Surgery 21.5 (1997): 529-533.

24. Rutala, William A., and David J. Weber. "Disinfection and sterilization in health care facilities: what clinicians need to know." Clinical Infectious Diseases 39.5 (2004): 702-709. Al-Naser KH Int J Med Res Health Sci 2017, 6(6): 132-137

25. Sharma, Atul K., Santosh Sharma, and Rakesh Sharma. "Port site infection in laparoscopic surgeries." Indian Medical Gazette (2013): 224-229

26. Espada, Mercedes, et al. "Insulation failure in robotic and laparoscopic instrumentation: A prospective evaluation." American Journal of Obstetrics and Gynecology 205.2 (2011): 121-e1.

27. Jacobs, P., and R. Kowatsch. "Sterrad sterilization system: A new technology for instrument sterilization." Endoscopic Surgery and Allied Technologies 1.1 (1993): 57-58. 